\title{
Using Enclosed Y-Mazes to Assess Chemosensory Behavior in Reptiles
}

\author{
M. Rockwell Parker ${ }^{*}$, , Andrea F. Currylow ${ }^{*}$, , Eric A. Tillman $^{3}$, Charlotte J. Robinson ${ }^{2}$, Jillian M. Josimovich ${ }^{2}$, Isabella M. G. \\ Bukovich $^{1}$, Lauren A. Nazarian ${ }^{1}$, Melia G. Nafus ${ }^{4}$, Bryan M. Kluever ${ }^{3}$, Amy A. Yackel Adams ${ }^{4}$ \\ ${ }^{1}$ Department of Biology, James Madison University ${ }^{2}$ U.S. Geological Survey, Fort Collins Science Center ${ }^{3}$ U.S. Department of Agriculture, National \\ Wildlife Research Center ${ }^{4}$ U.S. Geological Survey, Fort Collins Science Center \\ *These authors contributed equally
}

\section{Corresponding Author}

M. Rockwell Parker

mrockwellparker@gmail.com

\section{Citation}

Parker, M.R., Currylow, A.F.,

Tillman, E.A., Robinson, C.J.,

Josimovich, J.M., Bukovich, I.M.G.,

Nazarian, L.A., Nafus, M.G.,

Kluever, B.M., Adams, A.A.Y. Using

Enclosed Y-Mazes to Assess

Chemosensory Behavior in

Reptiles. J. Vis. Exp. (170), e61858,

doi:10.3791/61858 (2021).

\section{Date Published}

April 7, 2021

DOI

$10.3791 / 61858$

URL

jove.com/video/61858

\section{Abstract}

Reptiles utilize a variety of environmental cues to inform and drive animal behavior such as chemical scent trails produced by food or conspecifics. Decrypting the scenttrailing behavior of vertebrates, particularly invasive species, enables the discovery of cues that induce exploratory behavior and can aid in the development of valuable basic and applied biological tools. However, pinpointing behaviors dominantly driven by chemical cues versus other competing environmental cues can be challenging. Ymazes are common tools used in animal behavior research that allow quantification of vertebrate chemosensory behavior across a range of taxa. By reducing external stimuli, Y-mazes remove confounding factors and present focal animals with a binary choice. In our Y-maze studies, a scenting animal is restricted to one arm of the maze to leave a scent trail and is removed once scent-laying parameters have been met. Then, depending on the trial type, either the focal animal is allowed into the maze, or a competing scent trail is created. The result is a record of the focal animal's choice and behavior while discriminating between the chemical cues presented. Here, two Ymaze apparatuses tailored to different invasive reptile species: Argentine black and white tegu lizards (Salvator merianae) and Burmese pythons (Python bivittatus) are described, outlining the operation and cleaning of these $\mathrm{Y}$-mazes. Further, the variety of data produced, experimental drawbacks and solutions, and suggested data analysis frameworks have been summarized.

\section{Introduction}

Y-mazes are common, simple tools in studies of animal behavior that allow for a variety of questions to be addressed. In addition to being widely used in laboratory studies, $\mathrm{Y}$ - mazes are also functionally compatible with various field environments to study wild animals in relatively remote settings. Researchers have examined the behaviors of wild 
vertebrates using Y-mazes in a wide variety of taxa across similarly diverse field applications (e.g., lampreys ${ }^{1}$; cichlid fish $^{2}$; poison frogs ${ }^{3}$; lacertid lizards ${ }^{4}$; garter snakes ${ }^{5}$ ).

Many researchers are focused on how and to what degree chemical cues drive animal behaviors in reproductive, spatial, and foraging ecology ${ }^{6}$. A variety of chemical stimuli can be tested in Y-mazes and at fine scales, such as two chemical trails that only differ slightly in concentration ${ }^{7}$, or detection ability based on the reproductive status of the target species ${ }^{8}$. Chemical trails-the principal stimulus used in Y-maze tests-can be naturally created by conspecifics or specifically placed in the environment by a researcher using a defined chemical source ${ }^{1,5}$. Stimuli can also be tested in unique combinations to determine multimodal influence of cues such as changing contexts of cue presentation (airborne vs. substrate trails ${ }^{9}$; visual plus chemical cues $\left.{ }^{10}\right)$. Although there are many other methods for assessing chemosensory responses in reptiles (see discussion section), Y-mazes allow for searching behavior(s) to be assessed and at multiple temporal and spatial scales, which can lead to greater levels of behavioral inference.

Reptiles have been broadly tested for their reliance on chemical cues in reproductive and foraging ecology, and researchers often employ $\mathrm{Y}$-mazes in these studies ${ }^{11,12}$. The chemical ecology of reptiles continues to be deciphered by studies employing $\mathrm{Y}$-mazes to address a variety of evolutionary and behavioral questions that are valuable to wildlife managers. For example, recent tests with invasive snake and lizard species have revealed that chemical cues alone can influence choice and time allocation within the novel environment of a Y-maze ${ }^{13,14,15}$.

The use of large Y-mazes for moderately sized focal animals (e.g., large-bodied reptiles) is generally restricted to laboratory settings where the focal animals can be housed easily over the long term, experimental factors (e.g., climate, light, external stimuli) can be controlled, and access to infrastructure (e.g., power, running water) is unlimited. Studies on wild animals, however, are often restricted to specific locations for various reasons (e.g., logistics, permitting). As a result, challenges arise that must be addressed through creative problem solving and methodological adjustments to maintain consistent and comparable results.

Here, two experimental setups have been described using $Y$ mazes and remote monitoring tools to assess reproductive chemical ecology of invasive squamate reptiles (i.e., snakes and lizards) in different field scenarios: wild-caught, captive Argentine black and white tegu lizards (Salvator merianae) in Gainesville, FL, and wild-caught Burmese pythons (Python bivittatus) in Everglades National Park, FL. As implied by its name, the $\mathrm{Y}$-maze apparatus creates an experimental environment in which an animal enters a main passageway (the base of the $\mathrm{Y}$; "base") which then leads to two divergent passageways (the arms of the $Y$; "arms"). In these experiments, two types of animals are used for a single trial: scent-laying animals (provide the stimulus scent in a restricted area of the maze) and focal animals (data are collected on this animal as it explores the scent trail).

As an experimental apparatus in chemoecological studies, any $\mathrm{Y}$-maze must be constructed in a way that allows easy removal of the animal within and can be dissembled for thorough cleaning and reset. Also discussed are the constraints inherent to these different testing environments (e.g., diurnal vs. nocturnal animals, infrastructure differences) that prompted methodological adjustments. Although the focus was on tegu lizards and Burmese pythons, these 
designs can be applied to a wide range of reptile species. In this research on invasive reptiles, Y-mazes benefit the rate and scale of inference because they enable rapid collection of data to inform management goals that shift in-step with the invasion threat posed by a given species. In particular, studying chemoecology of invasive species is critical for the development of effective chemical control tools.

Discrimination is the key observation from empirical tests using Y-mazes where a focal animal chooses between two stimuli and that decision-making process is assessed. A swath of behaviors can also be scored in Y-maze trials during the trial itself (live) or after the trial (video) to expand inferential power. The complexity of the a priori objectives of a given study dictate whether live observation or archived recordings best suit the design. Here, Y-maze methods have been described in detail for addressing chemoecological questions to inform future studies by researchers interested in similar questions on reptile behavior, especially in chemical ecology.

\section{Protocol}

All procedures involving the use of live vertebrates were approved by the Institutional Animal Care and Use Committees of the U.S. Department of Agriculture and the U.S. Geological Survey.

NOTE: Because these studies focus on invasive vertebrates, compliance with containment standards must also be met, which impose specific constraints on the design and execution of experiments. Although many of the methods are similar between the two study locations and diurnal vs. nocturnal study timing, distinct methods have been described in each of the following two sections.

\section{Y-maze setup and diurnal protocol for the} U.S. Department of Agriculture (USDA) Animal
Plant Health Inspection Service (APHIS) Wildlife Services National Wildlife Research Center Florida Field Station: on-site testing of wild-caught, captive tegus

NOTE: Plans for all components of the Y-maze and containment structure are provided in Supplemental File 1.

1. Y-maze dimensions and design

1. Use a bottom piece $(1.22 \mathrm{~m} \times 2.44 \mathrm{~m}$ fiber cement siding panels) to anchor the Y-maze. Drill holes in the top layer to allow carriage bolts to pass upward for the attachment of the maze pieces. For specific directions, see Supplemental File 1.

2. Construct the walls of the maze out of white PVC trim board; internal dimensions of the base are 120 $\mathrm{cm} \mathrm{L}$ (side walls) $\times 42 \mathrm{~cm} \mathrm{~W} \times 14 \mathrm{~cm} \mathrm{H}$.

NOTE: Passageway width was designed to accommodate $2 x$ focal animal width. Extra width allows flexibility for two, animal-deposited scent trails to be created.

3. Make sure that the internal dimensions of the arms are $120 \mathrm{~cm} \mathrm{~L}$ (side walls) x $40 \mathrm{~cm} \mathrm{~W} \mathrm{x} 14 \mathrm{~cm} \mathrm{H}$.

4. Assemble the maze using separate bottom, side, and top components secured together prior to running a trial. Make the top out of clear acrylic to enable visualization of animals within maze. For specific directions, see Supplemental File 1.

1. When a single scent trail is created, use an internal partition in the base to restrict space access for the scent-laying tegu. For specific directions, see Supplemental File 1.

2. When two scent trails are deposited by different animals in sequence, use a system of partitions to block alternating arms of the 
maze and exclude each animal from alternating halves of the base. For specific directions, see Supplemental File 1.

5. Use boxes to allow transport and collection of animals used in Y-maze trials. Make sure that all boxes are opaque and fitted with removable lids and acrylic doors that are easily secured.

1. Ensure that the base box $(109 \mathrm{~cm} \mathrm{~L} \times 56 \mathrm{~cm}$ W $\times 46 \mathrm{~cm} \mathrm{H}$ ) is at the opening of the base of the Y-maze. Use it to transfer scenting or focal animals to the maze and for acclimation prior to opening the door and permitting voluntary access of the animals into the maze.

2. Make sure that the arm boxes $(83 \mathrm{~cm} \mathrm{~L} \times 50 \mathrm{~cm}$ W $\times 44 \mathrm{~cm} \mathrm{H}$ ) are at the terminal ends of the arms of the Y-maze to facilitate the capture of either scenting or focal animals.

3. For specific directions on construction and assembly, see Supplemental File 1.

2. Camera setup for diurnal video acquisition

1. Camera specifications: Ensure that project cameras can record continuous video under variable light conditions and are suitable for outdoor use under prevailing temperature and humidity conditions.

1. With the camera mounted to the underside of the study enclosure, ensure that the entire $\mathrm{Y}$ maze can be captured in the camera's field of view. Adjust the lens or height of the camera to increase or decrease the field of view. When the field of view is set, ensure that sufficient behavioral detail, such as tongue-flicks, can be captured.
NOTE: If the height of study enclosure is fixed (e.g., $180 \mathrm{~cm} \mathrm{H}$ ) thereby limiting adjustments to the field of view, multiple cameras can be used to obtain complete coverage of the Y-maze interior. Ensure that cameras are set to enable "wide dynamic range" when used in outdoor applications.

2. Power specifications: Ensure that each camera has an adequate power supply to record continuous video for the duration of the planned trial (e.g., use an Uninterruptible Power Supply (UPS) with a builtin backup battery to ensure continuous power).

NOTE: If there is no $A C$ power supply available, POE (power over ethernet) cameras can be powered via network cables connected to a digital video recorder (DVR) or POE switch where a network video recorder (NVR) is used.

3. Recording specifications: When choosing a DVR or $\mathrm{NVR}$, make sure that it meets project requirements including sufficient storage capacity and enough POE connectors (DVR) or camera channels (NVR) to accommodate the number of cameras used. Select recording parameters to suit the video quality desired, keeping the size of the data files in mind (e.g., H264 compression rate and an image rate of 10 frames per second [FPS]).

4. Protocol for obtaining and processing video:

1. Begin recording the video from the moment the animal begins to enter maze to the moment of capture or preset timeframe (1.3.3.4).

2. Using software that can be installed on one or more computers and allows viewing of live 
or recorded video, export files using the video format of choice.

3. Be sure to export the same time window and duration of video for each camera used to allow simultaneous review of multiple feeds.

4. Be sure to export the data regularly because many systems will overwrite older data with new data if DVR/NVR file storage capacity is limited.

3. Protocol for running scent-laying animals

1. Assessment of bias

1. Prior to running experimental trials, assess a Y-maze for bias by assembling the maze, as described below, but without presenting the scent on the paper. Acclimate the focal animal, and start the trial.

NOTE: Depending on the design of the study (e.g., repeated measures using the same focal animals vs. testing of novel focal animals each time), bias trials will establish that the maze itself, by design, does not bias a focal animal's choice. Many factors contribute to bias such as elevation, sunlight, and visual markers.

2. If reorienting or adjusting other physical aspects of the maze do not remove side bias, randomize the arm designated to receive an experimental scent in a given trial.

NOTE: Over a set number of trials, an unbiased maze results in a choice probability of 0.5 for either arm, and a binomial test is performed (Figure 2).

2. Trial preparation and Y-maze assembly
1. Wear nitrile gloves throughout when handling any surfaces that the animal can explore to avoid scent contamination. Change the gloves between trials and within the setup of a trial if multiple scent trails are being created.

2. Prepare new, clean scenting paper (white butcher paper, minimum $61 \mathrm{~cm}$ wide) on a clean surface. Cut to appropriate length such that the paper for each section can overlap at the junction of the $Y$ and extend past the ends of the base and the arms to fit under the boxes.

3. Sweep the bottom of the maze and then cover either with paper directly or with a boundary layer between the paper and bottom (e.g., plastic sheeting) to facilitate clean-up if the animal defecates or musks in the maze.

4. Secure the paper in place by piercing it with carriage bolts in the bottom, working from one end to another to keep the surface smooth. Overlap the papers at the junction such that the base paper is on top.

5. Place the sides of the maze into position over the carriage bolts, but do not secure them to the bottom.

6. Insert and secure the partition(s) needed for the trial type to be tested (see single-scent 1.3.3 vs. double-scent trials 1.3.4).

7. Slide the acrylic top pieces into slots, and secure with flat head nails.

8. Secure the sides to the bottom by tightening the wing nuts to the carriage bolts. 
9. Put the clean arm boxes into place, and secure with thumb screws. Secure the box lid using cable ties. Ensure that the doors have been removed.

3. Single-scent trials

NOTE: The purpose of these trials is to present a single scent trail in the $\mathrm{Y}$-maze that runs from the base through one arm.

1. Before fitting the acrylic top, secure the partition to block the untreated arm. Select the scented arm randomly (e.g., coin toss, random number generator).

2. Place the scent-laying animal into the clean, dry base box. Secure the lid of the base box (e.g., cable ties, bolts) and the door (e.g., thumb screw). Transport the holding box to the study enclosure, and secure it to the end of the base of the $Y$-maze with thumb screws.

NOTE: Ensure that the door to the base box is in place prior to loading the animal.

3. Acclimate the animal in the box for a set, consistent period (e.g., $60 \mathrm{~min}$ ). Remove the base box door, and allow the animal to enter the maze freely.

4. Monitor animal activity remotely using video feed (see below). After the animal has traveled from the base box to the arm box, remove the animal from the maze as scenting is complete.

1. If the animal is inside any box, insert and secure the removable door, remove the box, and return the animal to its enclosure.
2. If the animal is back in the maze, wait near the maze until the animal is seen returning to the box, and then remove the box.

NOTE: Squamates defecate defensively and create alarm cues that contaminate the scent being tested, so avoid startling the animal.

3. If the animal does not return to a box, slowly approach the maze and use visual cues (e.g., slow hand waving) to encourage the animal into the box, and then remove the box.

5. Clean and dry the base box (1.5.5).

6. If defecation occurred, collect and absorb as much as possible with a paper towel, but do not wipe to prevent spreading.

7. Partially disassemble the maze to allow removal of the interior partition, and then reassemble. Clean the partition (1.5.5).

8. Proceed to section 1.4 for protocol for running focal animals.

4. Double-scent trials

NOTE: The purpose of these trials is to present two different scent trails simultaneously in the $\mathrm{Y}$ maze, with both running from the base through their respective, randomly chosen arm.

1. Before fitting the acrylic top, secure the partitions to block the arm not chosen for the first scent and half of the base opposite to the blocked arm.

2. Follow the procedures outlined above for a single scent trial (1.3.3 to 1.3.3.8) with one 
exception. When the acrylic door is removed (1.3.3.3), insert a half-size door into the opening on the side that is to remain blocked to ensure that the scenting animal can only move in the open section of maze.

3. Partially disassemble the maze, remove the partitions, and clean (1.5.5). Dry with clean towels.

4. Reinstall the partitions, but flip them to block the now-scented area of the maze. Reinstall the acrylic top.

5. Repeat step 1.3.4.2 for the second scent-laying animal.

6. Partially disassemble the maze and remove the partitions. Reassemble the maze.

7. Proceed to section 1.4 for protocol for running focal animals.

4. Protocol for running focal animals during diurnal hours

1. Follow steps 1.3.3.2 to 1.3.3.3 with the focal animal planned for that trial.

2. Monitor animal activity remotely using video. If observing over a set window of exploration time, start the timer when the animal has completely emerged from the base box.

3. At the completion of the trial, remove the animal (1.3.3.4).

5. Breakdown and clean-up

1. Detach the remaining boxes from the maze and disassemble all the boxes. Wear fresh nitrile gloves throughout the disassembly and cleaning.
2. Remove the acrylic top pieces and set them aside in a safe location for cleaning to avoid scratching or cracking. Be sure to avoid scratching pieces when removed (clear field of view for video-monitoring behavior must be maintained). Disassemble the sides of the maze and set them aside for cleaning. NOTE: Minimize scratches and UV degradation to Y-maze materials by keeping them always shaded.

3. Remove the paper (and plastic) in a consistent motion by rolling it up to avoid contamination of the bottom and discard it.

4. Use an odorless, laboratory-grade soap and soft scrub brush or microfiber cloths to clean all the surfaces of the Y-maze pieces and all the boxes. Clean the acrylic top pieces and removable doors with the same soap, but with soft sponge or microfiber cloths to prevent scratching.

NOTE: The known chemical signals in squamate reptiles are lipid-soluble compounds, and washing with a detergent is the standard protocol for cleaning lipid cues and other scents from polymer-based apparatuses in terrestrial vertebrate studies ${ }^{11,12,21}$.

NOTE: In field applications, sanitation protocols may be required. If so, spray all the inner surfaces of the maze (floor, walls, partitions, acrylic pieces, boxes) with an appropriate sanitation solution, let it sit for 10 min, and then wipe with a microfiber cloth.

5. Rinse the cleaned components with water by wiping the surfaces with clean, wet microfiber towels, and avoid allowing soap residue to dry prior to rinsing; do not pour water into the maze. 
6. Allow the pieces to air dry or pat them dry with fresh microfiber cloths.

7. Once dry, reassemble the maze pieces if running another trial immediately.

\section{Y-maze setup and crepuscular timing protocol for the U.S. Geological Survey (USGS) trials in collaboration with National Park Service: relatively remote testing of wild-caught Burmese pythons}

NOTE: Plans for all components of the Y-maze and containment structure are provided in Supplemental File 2.

1. Y-maze components and rationale for changes to USDA design

NOTE: The Y-maze described was significantly altered to expand potential research species and in isolated conditions. The vertical depth was increased to accommodate a variety of species, and different materials and construction methods were used to improve outdoor durability and cleaning. See Figure 1 for a visualization of the completed maze. For specific directions on construction and assembly, see Supplemental File 2.

1. Cut Y-maze components from white polypropylene, and heat-weld all cut pieces that are to be permanently fixed (e.g., maze bottom and side walls).

1. Anchor the bottom of the $\mathrm{Y}$-maze $(244 \mathrm{~cm} \mathrm{~L} \mathrm{x}$ $122 \mathrm{~cm} \mathrm{~W}$ ) made of plywood sheets fastened together with deck screws, attaching it via an aluminum angle bracket riveted along the bottom of the outer side walls of the maze.

2. Ensure that the base of the $\mathrm{Y}$-maze is $120 \mathrm{~cm} \mathrm{~L}$ x $42 \mathrm{~cm} \mathrm{~W} \mathrm{x} 23 \mathrm{~cm} \mathrm{H}$, and that each outer arm side wall is $120 \mathrm{~cm} \mathrm{~L}$, inner arm side wall is 108 $\mathrm{cm} \mathrm{L} \mathrm{(for} \mathrm{specific} \mathrm{directions,} \mathrm{see} \mathrm{Supplemental}$ File 2).

3. Slot the acrylic top into place using an aluminum angle fixed to the side walls with screws every $30 \mathrm{~cm}$ (for specific directions, see Supplemental File 2).

NOTE: Screws placed at regular intervals at the top edge of the Y-maze side walls also serve as static visual markers when analyzing the video from the trials and provide scale.

4. Ensure that each opening of the $\mathrm{Y}$-maze (base, arms) has an additional baseplate $(42 \mathrm{~cm} \mathrm{~W}$ $x 30 \mathrm{~cm} \mathrm{H}$ ) on the end of the side wall that attaches to a box so that the baseplate frames a central opening ( $34 \mathrm{~cm} \mathrm{W \times 16} \mathrm{cm} \mathrm{H}$; for specific directions, see Supplemental File 2).

5. Use a partition piece to restrict access of the scenting animal (for specific directions, see Supplemental File 2). Secure a blocking plate $(46 \mathrm{~cm} \mathrm{~W} \times 22 \mathrm{~cm} \mathrm{H})$ in place using fastener tape. Anchor the partition and the plate using improvised, easily cleaned weights (e.g., plastic jug filled with water; 2.3.6; Figure 1).

6. Ensure that the acrylic pieces make up the top of the maze $(0.6 \mathrm{~cm}$ thick, clear). For specific directions, see Supplemental File 2.

2. Use opaque boxes fitted with a sliding door and lids that are easily secured to allow for transport and collection of animals in Y-maze trials (Figure 1).

1. Modify the boxes $(21.6 \mathrm{~cm} \mathrm{~L} \times 27.9 \mathrm{~cm} \mathrm{~W})$ with drain holes in the bottom, fit the lids with small screws and nuts, and provide a 
single opening for ingress/egress (the door). For specific directions, see Supplemental File 2.

2. Fasten the box to the end of the Y-maze by attaching the box faceplate to the $\mathrm{Y}$-maze faceplate using bolts and wingnuts or locks.

NOTE: When in place, boxes also anchor the acrylic top pieces in place.

2. Camera setup for crepuscular video acquisition: See Figure 1 for a snapshot of the camera field of view.

1. Camera specifications: Ensure that the project camera can record continuous video under variable light and temperature conditions to accommodate crepuscular and nocturnal study species.

1. With the project camera mounted on the ceiling crossbeams of the enclosure, ensure that the entire Y-maze can be captured within the camera's field of view. Raise or lower the height of the tent to increase or decrease the field of view (project camera mounted at a height of $\sim 3$ $m)$. Ensure that the reflection of the infrared light emitted from the camera on the acrylic top does not obscure critical portions of the frames in the overnight footage.

2. Power specifications: Ensure that each project camera has an adequate power supply to record continuous video for overnight filming (approximately $20 \mathrm{~h}$ ).

NOTE: If there is no AC power supply available, power can be supplied using deep cycle sealed lead acid 12-volt batteries (e.g., two 12-V 20-Ah gel batteries wired in parallel).

3. Recording specifications: To minimize the file storage volume, record the lowest quality video that is still adequate to enable counting of tongue-flicks in the $\mathrm{Y}$-maze.

NOTE: High-resolution footage requires large storage volume, and lowering the resolution is a very effective way of ensuring that file sizes are manageable.

1. Limit the framerate (frames per second, FPS) of footage to the minimum needed to detect tongue-flicks (e.g., recording resolution of 800 x 450 with a maximum framerate of 25 FPS results in approx.120 GB of footage per trial).

4. Protocol for obtaining and processing video

1. Arm the camera at the beginning of each scenting event (2.3.10), and let it record continuously through to the end of the focal event (approximately $20 \mathrm{~h}$ ).

2. After each trial is complete, power off the camera and retrieve the SD card (2.4.4). Transfer the footage to the desired storage location.

3. As SD cards frequently force recording devices to record footage in 5-min clips, combine these clips using movie processing software for ease of processing.

4. Review the footage using a media file reviewing program that allows variable playback speed and customizable forward-jump intervals.

NOTE: This reduces review time from about $20 \mathrm{~h}$ down to a maximum of $1 \mathrm{~h}$ if finescale resolution is not required during video processing.

3. Protocol for running scent-laying animals 
NOTE: Steps in this section will take approximately 1.5 days to complete due to longer acclimation times for wild reptiles.

1. See bias preface in section 1.3.1 to ensure that no bias can be found in the maze.

2. Wear nitrile gloves throughout when handling any surfaces or study animals to avoid scent contamination.

3. Place the scenting or focal animal into its box at least $24 \mathrm{~h}$ prior to the trial for acclimation.

NOTE: To minimize stress effects, the box is left in a shaded area as close to the maze as possible without being disturbed by cleaning or other activities. Ensure that all animals tested (scenting, focal) are acclimated this way.

4. Prepare new, clean scenting paper on a clean surface and of sufficient length to overlap at the junction of the $Y$, and cover the entire bottom surface $(2$ arm papers $=121.9 \mathrm{~cm} ; 1$ base paper $=152.4 \mathrm{~cm})$.

5. Secure the ends of the papers near the boxes and the $\mathrm{Y}$-junction with masking tape.

6. Install the partitions to block half of the base arm (left or right side) with a long partition, and block the entrance to the opposite arm with a short partition. When installing the barriers, do not rip the scenting paper. For large scenting animals, affix a heavy object that can easily be removed and cleaned behind the barrier as a brace to prevent barrier failure (2.1.1.5).

NOTE: The scent trail must always start on one side of the base, then cross to the opposite arm so that focal animal's choice is clear.
7. Slide the acrylic top into place, one section at a time, and ensure that the angles meet completely. Use clear plastic tape to cover any gaps.

8. Attach both the arm boxes to the maze by connecting the faceplates using wingnuts and/or padlocks, and ensure that the doors are locked open.

9. Two hours before sunset, attach the base box (containing the scenting animal), and ensure all movements are slow and steady to minimize stress to the animal.

10. Arm the camera, and open the door to the base box, being sure to latch the door in place using both barrel bolt locks. Remain out of the animal's view, and exit the area.

11. After $3 \mathrm{~h}$ ( $1 \mathrm{~h}$ after sunset), note the location of the animal within the maze as well as the ambient conditions. If the animal is in transit, wait until it enters the box.

1. If the animal is in any box, close and secure the box door, remove the box, and then remove the animal, taking care to prevent defensive scent deposition in the box.

2. If the animal is motionless inside the body of the maze, use visual cues (e.g., long rod or hand waving) to stimulate its movement into a box. If the animal remains, remove the arm box(es) so that the acrylic top can be removed, and the animal can be collected manually and transferred to a bag.

NOTE: Proper personal protective equipment (PPE) is always to be worn when handling large 
animals (e.g., puncture-resistant gloves, eye protection).

12. Partially disassemble the maze to allow removal of the interior partitions (avoid disturbing the scent paper) and then reassemble. If defecation occurred, collect and absorb as much as possible with clean microfiber cloths, but do not wash the area.

13. Proceed to section 2.4 for protocol for running focal animals.

4. Protocol for running crepuscular focal animals

NOTE: Steps in this section will take approximately 2 days to complete and must begin around the same time as the start of section 2.3.

1. Acclimate the scheduled focal animal in the box for at least $24 \mathrm{~h}$ prior to being run in maze.

1. During the final hours of focal animal acclimation, run the scent-laying animal prior to moving to the next step (2.3.9).
NOTE: Time the scent-laying step as close as possible to the time of introduction of the focal animal to the maze to reduce scent degradation.

2. Attach the base box (containing the focal animal) using wing nuts and/or padlocks to the base of the $Y$ maze. Use slow, steady movements when holding/ transporting the box to minimize stress to the focal animal.

1. Ensure that both the arm box doors are latched opened. Begin the focal trial by opening and latching the base box door using barrel bolts. Remain out of the animal's view and exit the area.

NOTE: With wild nocturnal reptile trials, focal animals are given overnight to explore the maze.

3. Four hours after sunrise, return to the maze and follow section 2.3.11.1 to remove the focal animal.

4. Collect the camera SD card and recharge the batteries if needed. Discard the used paper from the maze and proceed to cleaning (section 1.5). 

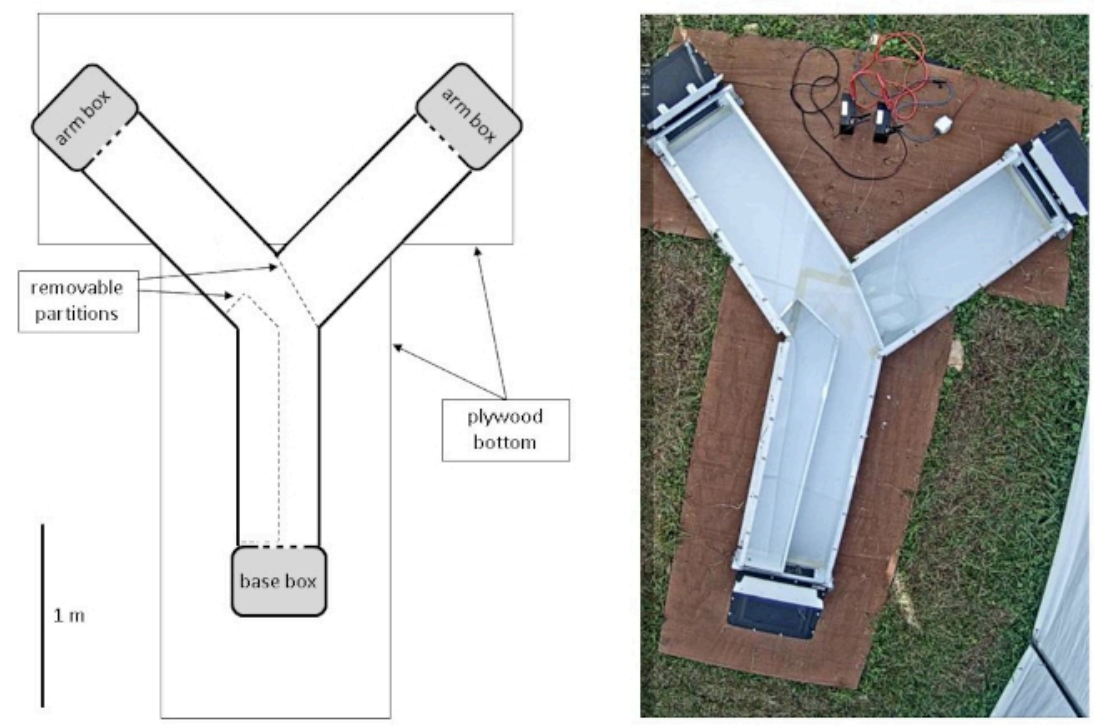

Figure 1. Layout of the USGS Y-maze. On the left, a schematic shows the components of the Y-maze with a scale bar for perspective. On the right, a snapshot from the video camera demonstrates the field of view for behavioral recordings. Please click here to view a larger version of this figure.

\section{Representative Results}

A multitude of variables can be recorded and/or scored from Y-maze trials. The design of the study should be primarily driven by the desired outcomes/deliverables. Further, if the study is relying on repeated measures (e.g., repeated use of the same focal animals), proper testing and analysis structures are required. For example, as the USDA trials relied on repeated testing of focal tegus, the planning of experimental trials was fully randomized.

Choice data: The majority of studies using Y-mazes report simple binary choice data and analyze the results with parametric statistics such as a binomial test. The chief limitation here is sample size, which directly affects the power of any statistical analysis. In Figure 2, a series of statistical thresholds per study sample size are depicted that demonstrate how many "successes" would need to occur for a given binomial test to yield statistically significant results. These are mathematically derived and therefore generalizable to any Y-maze test. Binomial statistics are easy to generate using online freeware. For calculating probabilities, one-tail distributions are used if an a priori rationale is given; otherwise, the two-tail distribution should be used.

Choice of an arm is often determined by the distance the focal animal moves in a given arm. The simplest way to set this threshold is by establishing a landmark within the maze. For most $\mathrm{Y}$-maze studies, the landmark is the entrance of the arm box. Because reptiles conduct all chemosensory assessment with the chemical-sensing organs in the anterior region of the head, the head is the focal point during a trial. For example, because Burmese pythons are often longer than the entire 
maze itself, choice is best and most efficiently determined by the movement of the head past a landmark. Other options for determining choice are time spent in an arm and complete movement of the focal animal into a box. Failure is determined by a focal animal not making a choice within a specific period.

More fine resolution analyses can be derived from choice data in the Y-maze. For example, researchers can generate a choice penalty score ${ }^{16}$. Here, researchers must track the degree to which the focal animal explored the non-target arm of the maze. Non-target can be defined as the arm the researchers determine a priori that the focal animal will not choose based on the alternative hypothesis tested. The simplest example of a non-target arm would be the unscented arm when only one arm contains a target scent. More complex examples would be the choice between two scents from the same source, but presented at different concentrations ${ }^{7}$. When the experimental design is multi-level and/or the data go from binary to incremental, as with choice penalty, an appropriate statistical approach should be used such as repeated measures analysis of variance (ANOVA) or other methods used with continuous or proportional datasets.

Behaviors: Throughout the duration of an experiment in which focal animals are observed, a variety of individual behaviors can be quantified. This number of variables can either be determined a priori depending on what is known $^{16}$ or post hoc following preliminary observations on a subset of data ${ }^{14,15}$. The study objectives and their degree of resolution determine what behavioral assessments should be made within the maze, if any (i.e., in many studies, only choice data are quantified ${ }^{17}$ ). Behaviors can be assessed throughout the maze, in sections, or during specific time periods; for instance, behaviors seen only in the base or at the junction of the arms may be prioritized ${ }^{8}$. Video recordings facilitate behavioral scoring, although the resolution of the video and its length-factors that impose data storage constraints-should be considered before experimentation begins.

Temporal variables: As with behavioral variables, many temporal aspects of animal performance can be quantified during Y-maze trials. For example, researchers can time latency periods (e.g., latency to emerge from the box ${ }^{8}$ ). Most temporal variables are associated with exploration of the maze such as total trailing time or time spent in each arm. These variables are usually analyzed in a multi-factor analysis such as multi-way ANOVA.

Observer bias: With any studies involving animal behavior, observer bias significantly influences data collection ${ }^{18}$. Therefore, observers should be blind to the treatment being tested. The simplest way to do this is to code the video files numerically and then randomly sort them (e.g., random number generator) prior to assigning them to observers. Controlling for observer bias is difficult-to-impossible when live data collection is the only option. In a field setting, this would require two cooperators: an observer blind to the treatment and a coordinator who sets up the trial. Extensive reviews summarize the effects of experimenter bias on data collection and interpretation in behavioral and ecological studies $^{18,19}$. 


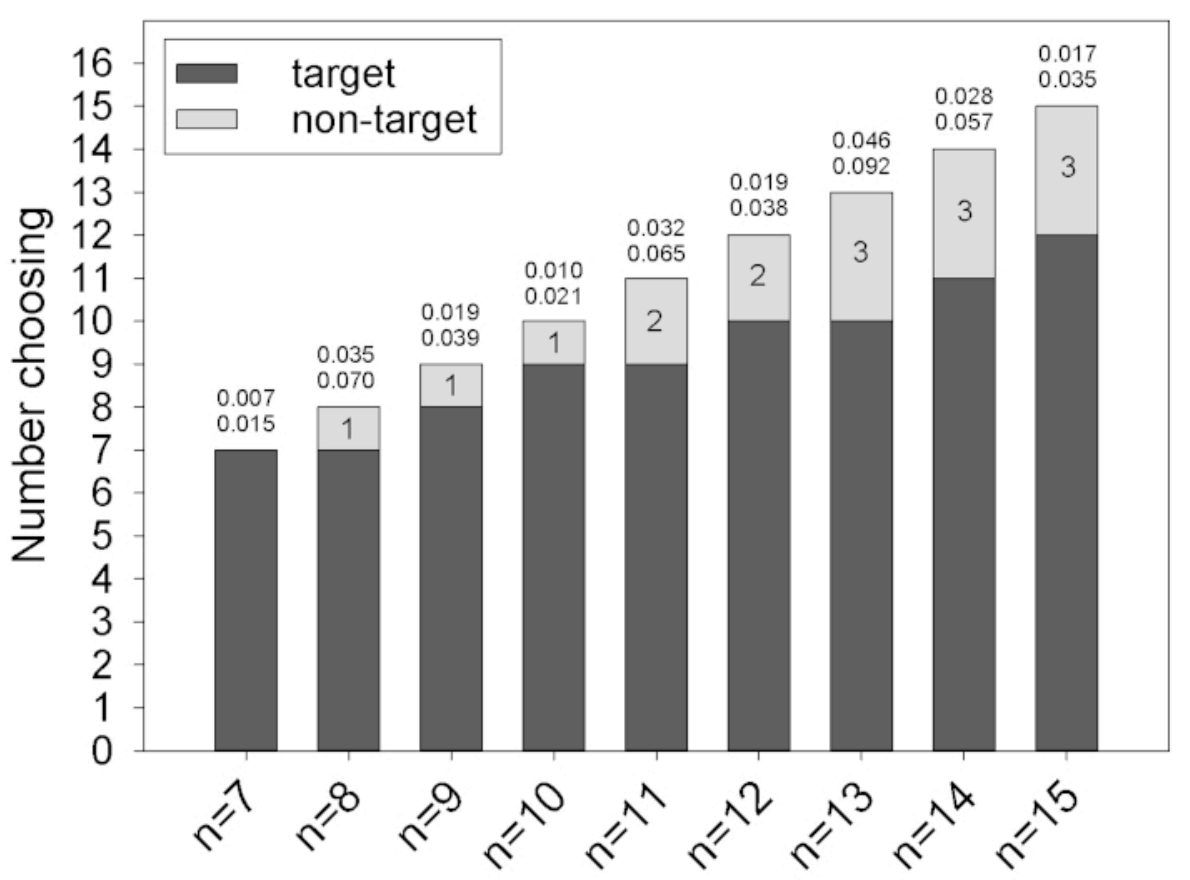

Figure 2. Sample sizes and P-values for binomial tests from Y-maze results. Each given sample size represents a set number of trials where a scent is tested in one arm of the $Y$ (target arm) while the other could be a control (non-target). Top number above each bar is the one-tail P-value for that number of target arm choices, bottom is two-tail. Numbers within the top bar represent the maximum number of non-target choices that are still traditionally statistically significant $(P<$ 0.05). Please click here to view a larger version of this figure.

Supplemental File 1. Please click here to download this file.

Supplemental File 2. Please click here to download this file.

\section{Discussion}

While Y-mazes are very powerful tools to investigate chemical ecology in reptiles, their limited design can preclude other avenues of inquiry. However, a diversity of other options is available $11,12,20,21,22$. For example, tongue-flick assays are simpler to execute and allow simultaneous assessment of behaviors exhibited to an array of chemical stimuli relative to control odors $23,24,25,26$. Open-field tests are another option where a focal animal freely explores an enclosure until it encounters a source of chemical cues, and its behavioral reactions are subsequently scored 27,28 . Combinations of these approaches can assess discriminatory capacities of reptiles in varying contexts such as presenting a mix of 
artificial and natural odors along with refugia ${ }^{29}$. Y-mazes can also be modified to expose animals to airborne chemical cues alone or in combination with substrate-borne cues ${ }^{16,30}$, and post hoc inference can be used to redesign data collection if archived video data are available ${ }^{31}$. Bioassays should be designed to simplify data collection and minimize conflicting stimuli, especially when a specific source of cues is being assessed (e.g., chemical cues ${ }^{21}$ ).

Researchers in animal behavior often observe and quantify focal animal responses in novel, artificial environments (e.g., an enclosed maze with a featureless landscape), and care should be taken to assess whether a given animal is exhibiting natural, exploratory behavior versus avoidance, agitation, or similar distressed behavior. Distressed animal behavior in experimental apparatuses is primarily attributed to neophobia: fear of novelty ${ }^{32}$. An example is escape behavior, where the focal animal pushes against the joints or the edges of the apparatus to achieve egress. Another example is shyness, where the focal animal demonstrates reluctance to enter the maze, the degree of which can be quantified by latency of maze entry. Apparatus (re)design can facilitate engagement of the focal animal to avoid these confounding effects of distress. The most common approach is repeated introduction of the focal animal to the apparatus to remove the novelty of the environment before testing begins, and contemporary statistical models (e.g., generalized linear mixed models) allow for test animals to be used in multiple trials. An important aside relevant to ecological considerations in behavioral testing is that reduced neophobia is associated with the success of invasive species $^{33}$. Thus, depending on a priori knowledge of the species in question, neophobia may have variable importance as an experimental design consideration.
Acquisition of behavioral data from videos imposes multiple constraints that become major bottlenecks in experimental timelines. For example, the length of a given trial can exponentially increase data extraction time. One workaround is to analyze behavior only until a threshold is met (e.g., total time active). The threshold can be based on the longest video available for a given trial. Alternatively, machine-based observation (e.g., artificial intelligence) can be developed, although this is time- and resource-consuming with considerable effort required for quality control. Another issue is data management: videos must be of sufficient quality to enable behavioral scoring and assessment, resulting in data storage constraints. While cloud storage is now accessible, upload/download rates are often problematic, especially when data acquisition occurs in remote field locations. Additional challenges manifest in the limitations of recording tools that affect the integrity of behavioral observation. Clear viewing of focal animal behavior is always necessary, but visibility is often impeded by uncontrollable factors (e.g., moisture, insects, wind movement). Further, when recordings come from a single perspective (e.g., bird's eye view), behaviors occurring in the vertical plane (e.g., head raises ${ }^{14}$ ) are difficult to assess. A solution is to provide multiple camera angles per trial. Lastly, the time of day significantly affects behavioral recording. Nighttime behavioral analysis requires a camera with a nighttime mode and minimal light projection to avoid obstructive glare on the Y-maze surface or attraction of insects that can interrupt the camera feed. Considering the above, foreknowledge of the study site or species biology can inform which constraints are likely to occur with what frequency and thus inform desirable sample sizes. 
Behavior is tightly coupled with physiology, and the utility of Y-mazes for evaluation of behavioral endocrinology in a variety of species has been demonstrated. However, this paper emphasizes some variation in the execution of these experiments depending on the target species, research question, and resources available. Therefore, the selection of materials and dimensions of each testing setup should be carefully considered for potential subsequent research expansion. Section 2 describes modifications made to materials outlined in section 1, which were incorporated to accommodate future, more complex behavioral trials with tegus. The increased vertical depth of the Everglades mazes will allow new questions about chemical ecology in wild-caught tegus to be answered without unduly protracting project design and setup, further demonstrating the translatability of this experimental apparatus.
When employing the above-described techniques in a relatively remote setting (see section 2), there are several limiting factors that must be considered, and project planning is paramount. Depending on the statistical power needed for the prescribed treatment experiment and biological timing of the target species (e.g., seasonality), the resources and labor required will be affected. Further, if single or repeated use of focal animals are desired, careful attention to reducing potential stressors is necessary. Each of these factors will either extend the project timeline or require increased labor, space, and materials. For example, section 2 presents the use of wild-caught male pythons as focal animals trailing another group of wild-caught and hormonally manipulated males, all of which require approximately $24 \mathrm{~h}$ of quiet acclimation time in holding boxes to minimize stress effects. Although these acclimation periods extended trial times to over two days, stress due to captivity and handling affect wild animal behavior and must be minimized to generate clean datasets $^{34,35}$.

In summary, Y-mazes are powerful, adaptable tools that can be used to investigate the chemical ecology of diverse wildlife under widely variable conditions, provided there is vigilant a priori planning. Careful consideration must be taken to choose appropriate questions and to properly design the experimental setup for given taxa and conditions. Researchers and managers can significantly benefit from using $\mathrm{Y}$-mazes to better understand animal chemosensory biology as these tools enable flexible experimental designs that provide large volumes of fine-scale behavioral data, especially when combined with remote monitoring tools.

\section{Disclosures}

None 


\section{Acknowledgments}

The development of the first $\mathrm{Y}$-maze was supported by cooperative agreements (15-7412-1155-CA, 16-7412-1269CA, and 17-7412-1318-CA) between James Madison University (JMU) and the USDA Animal and Plant Health Inspection Service. The development of the Y-maze in Everglades National Park was funded by a cooperative agreement (P18AC00760) between JMU and the National Park Service. We thank T. Dean and B. Falk for their facilitation of this project in Everglades NP and assistance with permitting and funding. We thank W. Kellow for assistance in construction of the USGS Ymaze. C. Romagosa, L. Bonewell, and R. Reed provided administrative and logistical support. We thank the two anonymous reviewers who offered helpful feedback. Funding for the Everglades work and in-kind support was provided by U.S. Geological Survey (USGS) Greater Everglades Priority Ecosystem Science Program, National Park Service (P18PG00352), and USGS Invasive Species Program. Any use of trade, firm, or product names is for descriptive purposes only and does not imply endorsement by the U.S. Government. The findings and conclusions in this publication have not been formally disseminated by the U.S. Department of Agriculture and should not be construed to represent USDA determination or policy.

\section{References}

1. Fine, J. M., Vrieze, L. A., Sorensen, P. W. Evidence that petromyzontid lampreys employ a common migratory pheromone that is partially comprised of bile acids. Journal of Chemical Ecology. 30 (11), 2091-2110 (2004).
2. Hesse, S., Bakker, T. C., Baldauf, S. A., Thünken, T. Kin recognition by phenotype matching is familyrather than self-referential in juvenile cichlid fish. Animal Behaviour. 84 (2), 451-457 (2012).

3. Forester, D. C., Wisnieski, A. The significance of airborne olfactory cues to the recognition of home area by the dart-poison frog Dendrobates pumilio. Journal of Herpetology. 25 (4), 502-504 (1991).

4. Khannoon, E. R., El-Gendy, A., Hardege, J. D. Scent marking pheromones in lizards: cholesterol and long chain alcohols elicit avoidance and aggression in male Acanthodactylus boskianus (Squamata: Lacertidae). Chemoecology. 21 (3), 143-149 (2011).

5. Parker, M. R., Mason, R. T. How to make a sexy snake: estrogen activation of female sex pheromone in male red-sided garter snakes. Journal of Experimental Biology. 215 (5), 723-730 (2012).

6. Wyatt, T. D. Pheromones and animal behavior: chemical signals and signatures. Cambridge University Press, (2014).

7. Smith, T. L., Bevelander, G. S., Kardong, K. V. Influence of prey odor concentration on the poststrike trailing behavior of the Northern Pacific Rattlesnake. Herpetologica. 61 (2), 111-115 (2005).

8. Yosuke, K., Akira, M. Active foraging for toxic prey during gestation in a snake with maternal provisioning of sequestered chemical defences. Proceedings of the Royal SocietyB: Biological Sciences. 282, 20142137 (2015).

9. Parker, M. R., Kardong, K. V. The role of airborne and substrate cues from non-envenomated mice 
during rattlesnake (Crotalus oreganus) post-strike trailing. Herpetologica. 62 (4), 349-356 (2006).

10. Bezzina, C. N., Amiel, J. J., Shine, R. Does invasion success reflect superior cognitive ability? A case study of two congeneric lizard species (Lampropholis, Scincidae). PLoS ONE. 9 (1), e86271 (2014).

11. Mason, R. T., Parker, M. R. Social behavior and pheromonal communication in reptiles. Journal of Comparative Physiology A. 196 (10), 729-749 (2010).

12. Parker, M. R., Mason, R. T. Pheromones in snakes: history, patterns and future research directions. In Reproductive Biology and Phylogeny of Snakes. (eds Robert D. Aldridge \& David M. Sever) 563-584 CRC Press, (2011).

13. Greene, M. J., Stark, S. L., Mason, R. T. Pheromone trailing behavior of the brown tree snake, Boiga irregularis. Journal of Chemical Ecology. 27 (11), 2193-2201 (2001).

14. Richard, S. A., Tillman, E. A., Humphrey, J. S., Avery, M. L., Parker, M. R. Male Burmese pythons follow female scent trails and show sex-specific behaviors. Integrative Zoology. 14 (5), 460-469 (2019).

15. Richard, S. A. et al. Conspecific chemical cues facilitate mate trailing by invasive Argentine black and white tegus. PLoS ONE. 15 (8), p.e0236660 (2020).

16. Parker, M. R., Kardong, K. V. Airborne chemical information and context-dependent post-strike foraging behavior in Pacific Rattlesnakes (Crotalus oreganus). Copeia. 105 (4), 649-656 (2017).

17. Lutterschmidt, D. I., Maine, A. R. Sex or candy? Neuroendocrine regulation of the seasonal transition from courtship to feeding behavior in male red-sided garter snakes (Thamnophis sirtalis parietalis). Hormones and Behavior. 66 (1), 120-134 (2014).

18. Burghardt, G. M.et al. Perspectives-minimizing observer bias in behavioral studies: a review and recommendations. Ethology. 118 (6), 511-517 (2012).

19. Holman, L., Head, M. L., Lanfear, R., Jennions, M. D. Evidence of experimental bias in the life sciences: why we need blind data recording. PLoS Biology. 13 (7), e1002190 (2015).

20. Mason, R. T. Reptilian pheromones. In Biology of the Reptilia: Hormones, brain, and behavior. Vol. 18 (eds Carl Gans \& David Crews) 114-228 University of Chicago Press, (1992).

21. Mason, R. T., Chivers, D. P., Mathis, A., Blaustein, A. R. Bioassay methods for amphibians and reptiles. In Methods in Chemical Ecology. Vol. 2 (eds Kenneth F. Haynes \& Jocelyn G. Millar) 271-325 Springer Science \& Business Media, (1998).

22. Martín, J., López, P. Pheromones and chemical communication in lizards. In Reproductive biology and phylogeny of lizards and tuatara. (eds Justin L. Rheubert, Dustin S. Siegel, \& Stanley E. Trauth) 43-77 CRC Press, (2014).

23. Smith, K. P., Parker, M. R., Bien, W. F. Behavioral variation in prey odor responses in northern pine snake neonates and adults. Chemoecology. 25 (5), 233-242 (2015).

24. Parker, M. R., Patel, S. M., Zachry, J. E., Kimball, B. A. Feminization of male Brown Treesnake methyl ketone expression via steroid hormone manipulation. Journal of Chemical Ecology. 44 (2), 189-197 (2018). 
25. Cooper, W. E. Evaluation of swab and related tests as a bioassay for assessing responses by squamate reptiles to chemical stimuli. Journal of Chemical Ecology. 24 (5), 841-866 (1998).

26. Goetz, S. M., Godwin, J. C., Hoffman, M., Antonio, F., Steen, D. A. Eastern indigo snakes exhibit an innate response to pit viper scent and an ontogenetic shift in their response to mouse scent. Herpetologica. 74 (2), 152-158 (2018).

27. Clark, R. W. Timber rattlesnakes (Crotalus horridus) use chemical cues to select ambush sites. Journal of Chemical Ecology. 30 (3), 607-617 (2004).

28. Martín, J., López, P. Supplementation of male pheromone on rock substrates attracts female rock lizards to the territories of males: a field experiment. PLoS ONE. 7 (1), e30108 (2012).

29. Downes, S., Shine, R. Sedentary snakes and gullible geckos: predator-prey coevolution in nocturnal rockdwelling reptiles. Animal Behaviour. 55 (5), 1373-1385 (1998).

30. Parker, M. R., Kardong, K. V. Rattlesnakes can use airborne cues during post-strike prey relocation. In Chemical Signals in Vertebrates 10. (eds Michael P. LeMaster, Robert T. Mason, \& Dietland MüllerSchwarze) 397-402 Springer, (2005).

31. Parker, M. R., Young, B. A., Kardong, K. V. The forked tongue and edge detection in snakes (Crotalus oreganus): an experimental test. Journal of Comparative Psychology. 122 (1), 35-40 (2008).

32. Greggor, A. L., Thornton, A., Clayton, N. S. Neophobia is not only avoidance: improving neophobia tests by combining cognition and ecology. Current Opinion in Behavioral Sciences. 6, 82-89 (2015).

33. Candler, S., Bernal, X. E. Differences in neophobia between cane toads from introduced and native populations. Behavioral Ecology. 26 (1), 97-104 (2015).

34. Currylow, A. F., Louis, E. E., Crocker, D. E. Stress response to handling is short lived but may reflect personalities in a wild, Critically Endangered tortoise species. Conservation Physiology.5 (1), cox008 (2017).

35. Currylow, A. F.et al. Comparative ecophysiology of a critically endangered (CR) ectotherm: Implications for conservation management. PLOS ONE. 12 (8), e0182004 (2017). 